\title{
A survey of Canadian urologists' opinions and prescribing patterns of testosterone replacement therapy in men on active surveillance for low-risk prostate cancer
}

\author{
Adam C. Millar, MD; ${ }^{1^{*}}$ Dean S. Elterman, MD ${ }^{2 *}$ Larry Goldenberg, MD; ${ }^{3}$ Brandon Van Asseldonk, BASc ${ }^{4}$ \\ Ashley Curtis, MA, ${ }^{4}$ Keith Jarvi, $M D^{5}$
}

'Division of Endocrinology and Metabolism, Department of Medicine, Mount Sinai Hospital, University of Toronto, Toronto, ON, Canada; 2Division of Urology, Department of Surgery, University Health Network, Toronto, ON, Canada; ${ }^{3}$ Department of Urologic Sciences, University of British Columbia Vancouver, BC, Canada; ${ }^{4}$ Division of Urology, Department of Surgery, University of Toronto, Toronto, ON, Canada; ${ }^{5}$ Division of Urology, Mount Sinai Hospital, Toronto, ON, Canada

*Dr. Millar and Dr. Elterman contributed equally as co-first authors.

Cite as: Can Urol Assoc J 2016;10(5-6):181-4. http://dx.doi.org/10.5489/cuaj.3608

See related commentary on page 185.

\section{Abstract}

Introduction: Attitudes regarding the safety of testosterone replacement therapy (TRT) in hypogonadal men with prostate cancer (PCa) have changed over the past few years with the emergence of case studies suggesting a low risk of cancer progression and a better understanding of the interaction of different levels of androgen with prostate cellular metabolism. This new view has the potential to change clinical practice.

Methods: Active members of the Canadian Urological Association were surveyed about their opinions on the safety of TRT in men with low-risk PCa, as well as their current prescribing habits.

Results: Of 57 responding urologists, 86\% actively prescribe TRT in men with testosterone deficiency syndrome (TDS), 93\% are involved in the treatment of men with $\mathrm{PCa}$, and 95\% offer active surveillance as a management option for low-grade/low-stage disease. Furthermore, $65 \%$ stated that they would offer TRT to men with TDS who were on active surveillance for PCa and 63\% believed that TRT did not increase the risk of progression of PCa in these men. In terms of treatment methods, 96\% believed TRT was safe for men who have undergone radical prostatectomy, while a smaller number felt it was safe for patients who have undergone brachytherapy $(86 \%)$ or external beam radiation (84\%). Despite these figures, only $35 \%$ of the surveyed physicians had ever offered TRT for men on active surveillance and only $42 \%$ actually had men in their practice who were taking testosterone while on active surveillance.

Conclusions: The discrepancy between urologists' beliefs about the safety of TRT and their clinical practice patterns may be due to multiple factors, such as hesitation in recommending treatment in real-life practice, low numbers of eligible patients, absence of screening for testosterone deficiency in patients on active surveillance, and patient preference or fears. Furthermore, the difference in perceived safety in men treated by radical prostatectomy vs. radiation therapy suggests that some urologists are concerned that the radiated gland remaining in-situ may be "reactivated" by TRT. The results from this survey will be used as the basis of developing a national Canadian registry of men with low-grade/stage PCa who are receiving TRT while on active surveillance.

\section{Introduction}

Testosterone deficiency syndrome (TDS) occurs in approximately $20 \%$ of men aged 50 , with an increasing incidence with age. ${ }^{1}$ At the same time, prostate cancer (PCa) has an increasing prevalence with age. ${ }^{2}$ Hypogonadism in men carries a range of symptoms that can have significant impact on quality of life, including weakness, decreased libido, erectile dysfunction, and loss of muscle mass. Some clinical observational studies suggest that testosterone replacement therapy (TRT) can improve many of these symptoms. ${ }^{3}$

For decades, it has been taught that TRT in patients with PCa was dangerous. These beliefs were largely based on the Nobel prize-winning observations by Huggins and Hodges in 1941, showing regression of metastatic PCa following suppression of testosterone by either estrogen administration or castration. ${ }^{4}$ The same researchers suggested that as a corollary, the administration of testosterone would lead to growth of existing cancer. In their investigation of three patients with advanced PCa, they showed that acid phosphatase levels increased with testosterone injections and returned to baseline levels upon cessation of these injections. ${ }^{4} \mathrm{~A}$ few days after testosterone injection cessation, the levels of acid phosphatase of one patient increased sharply and persisted. ${ }^{4}$ For years thereafter, based on the results of this small sample, it was globally accepted that any history of PCa was an absolute contraindication for TRT in hypogonadal men. However, this has proven to be an inaccurate extrapolation. 
Six decades after these original experiments, new studies suggest that administration of TRT in selected groups of patients with PCa may not be dangerous, but in fact, potentially beneficial. Two separate studies that examined a total of 17 men with a history of radical prostatectomy and who were treated with TRT for TDS found no recurrence of cancer. ${ }^{5,6}$ A 2013 study by Pastuszak et al examined 152 men who underwent total prostatectomy for $\mathrm{PCa}^{7} 103$ hypogonadal men treated with TRT (26 of whom were "highrisk"), and a reference group of 49 non-hypogonadal men. They found an increase in prostate-specific antigen (PSA) in the treatment group, but only four of these men had cancer recurrence, compared to eight in the reference group. This is the largest study to date evaluating men with a history of PCa who are receiving TRT.

Sarosdy et al followed 31 men treated with brachytherapy and TRT with no recurrence noted after five years of followup. ${ }^{8}$ Morales et al reported on the treatment of five men post-external beam radiotherapy (EBRT) for $\mathrm{PCa}$, noting a small rise in PSA to less than $1.5 \mathrm{ng} / \mathrm{mL}$, but no evidence of cancer recurrence in any patient. ${ }^{9}$ Pastuszak et al retrospectively reviewed 13 men who underwent either EBRT or brachytherapy for PCa and were administered TRT for hypogonadal symptoms. ${ }^{10}$ There were no significant PSA increases or PCa progression, while improvement in hypogonadal symptoms was noted.

The use of TRT has been more controversial in men with low-risk PCa on active surveillance, because the cancer has been left untreated. Morgentaler et al retrospectively reviewed 13 men (Gleason score 6 in 12 patients; 7 in one patient) who were treated with TRT while on active surveillance for a median of 2.5 years. ${ }^{11}$ There was no progression of PCa.

The saturation theory of prostatic-androgen relationship has been proposed to explain the apparent dichotomy of castration being therapeutic while TRT in a hypogonadal (NOT castrate) man has no impact on cancer progression. This theory states there is a saturation point, or a threshold, at a relatively low level of serum testosterone above which there is no increase in the translocation of androgen into prostatic cellular nuclei, and subsequent activation of androgen receptors. ${ }^{12,13}$ Dose-response only occurs at very low levels of testosterone, likely near castrate range and not near the hypogonadal space.

Despite these well-documented observations, there remains uncertainty among many urologists about the safety of TRT in hypogonadal men who are either on active surveillance, or who have had curative therapy. There are presently no large, randomized, control trials to confirm the potential safety of testosterone in such patients. It therefore remains unclear how many physicians believe this to be true. Furthermore, it is not known how many physicians are actually prescribing TRT to such patients.
In this study, we surveyed active members of the Canadian Urological Association (CUA) to determine their attitudes, beliefs, and practice patterns regarding the care of hypogonadal men with known PCa.

\section{Methods}

A 10-item dichotomous (yes/no) questionnaire (Table 1) was designed to evaluate the attitudes, beliefs and practice patterns of physician members of the CUA. Ethics approval was obtained from the local institutional research ethics board. CUA members were contacted via two separate email letters and invited to participate in the questionnaire. Participation was voluntary and no compensation was provided. Responses were collected and stored on the SurveyMonkey ${ }^{\circledR}$ website. Responses were anonymous and no personal information was collected.

\section{Results}

Of 709 invited CUA members, $57(8 \%)$ responded to the questionnaire. The questionnaire items and complete results are shown in Table 1. Of these 57 physicians, 93\% actively treat men with PCa and 95\% offer active surveillance as a management option for men with low-grade/low-stage disease.

Notably, $86 \%$ of the surveyed urologists generally prescribe TRT for men with known testosterone deficiency without known PCa and $65 \%$ believe that it is safe for men with PCa on active surveillance to be treated with testosterone in the presence of testosterone deficiency. Furthermore, 63\% believe that use of testosterone did not increase the risk of progression of PCa in men on active surveillance. Finally, $65 \%$ stated that they would offer TRT for men with TDS who were on active surveillance.

Despite the above figures, however, only $35 \%$ of physicians had ever offered TRT for men on active surveillance and only $42 \%$ actually had men in their practice who were taking testosterone while on active surveillance.

Regarding men with treated $\mathrm{PCa}, 96 \%$ of physicians felt it was safe to administer testosterone to patients who underwent radical prostatectomy for PCa. In comparison, $84 \%$ of physicians felt it was safe after radiation therapy and $86 \%$ felt it was safe after brachytherapy.

\section{Discussion}

The current body of evidence supporting TRT in men with PCa is restricted to a few small clinical trials..$^{5-11}$ An important question is whether these observations are substantial enough to impact the clinical management of hypogonadism in men under active surveillance for low-stage/grade PCa. The evidence specifically pertaining to active surveillance 


\begin{tabular}{|c|c|c|}
\hline Questionnaire item & Total “Yes" respondents (\%) & Total "No" respondents (\%) \\
\hline 1. Do you treat men with $\mathrm{PCa}$ ? & $53(93 \%)$ & $4(7 \%)$ \\
\hline $\begin{array}{l}\text { 2. Do you use active surveillance as one method to } \\
\text { manage men with low-grade/low-stage PCa? }\end{array}$ & $54(95 \%)$ & $3(5 \%)$ \\
\hline $\begin{array}{l}\text { 3. Do you prescribe TRT for patients with TDS without } \\
\text { PCa? }\end{array}$ & $49(86 \%)$ & $8(14 \%)$ \\
\hline $\begin{array}{l}\text { 4. Is it safe to prescribe TRT for patients on active } \\
\text { surveillance?* }\end{array}$ & $36(63 \%)$ & $20(35 \%)$ \\
\hline $\begin{array}{l}\text { 5. Does TRT increase risk of PCa progression in men on } \\
\text { active surveillance?* }\end{array}$ & $20(35 \%)$ & $36(63 \%)$ \\
\hline $\begin{array}{l}\text { 6. Would you offer TRT for TDS if on active suveillance } \\
\text { for PCa? }\end{array}$ & $37(65 \%)$ & $20(35 \%)$ \\
\hline $\begin{array}{l}\text { 7. Have you offered TRT for men with TDS on active } \\
\text { surveillance? }\end{array}$ & $20(35 \%)$ & $37(65 \%)$ \\
\hline $\begin{array}{l}\text { 8. Do you have men on active surveillance who are on } \\
\text { TRT? }\end{array}$ & $24(42 \%)$ & $33(58 \%)$ \\
\hline \multicolumn{3}{|l|}{$\begin{array}{l}\text { 9. Is it safe to prescribe TRT for men who received } \\
\text { curative treatment for PCa using the following: }\end{array}$} \\
\hline a) Radical prostatectomy & a) 55 (96\%) & a) $2(4 \%)$ \\
\hline b) Radiation therapy** & b) $48(84 \%)$ & b) $8(14 \%)$ \\
\hline c) Brachytherapy & c) $49(86 \%)$ & c) $8(14 \%)$ \\
\hline
\end{tabular}

is even more limited, but the results of our survey reveal that $65 \%$ of a representative sample of Canadian urologists believe that this practice is safe. One could argue that the historical evidence that initially triggered the fear of TRT in men with PCa was based on an even smaller cohort (only three patients), but time and widespread teaching have fortified this belief. It is clear that many urologists are persuaded by the new evidence, although there remains a proportion that are not yet comfortable or convinced of the absolute safety. Therefore, randomized clinical trials with larger sample groups are necessary to provide substantial evidence that TRT does not lead to progression of PCa in patients under active surveillance.

In regard to men previously treated with radical prostatectomy or radiation therapy, our survey reveals that almost all urologists are prepared to give a post-surgical patient TRT, but 10-12\% fewer are comfortable doing the same for radiated patients. This may be due to a level of uncertainty when prostate tissue remains in vivo.

Despite completing two phases of data collection (two separate emails), the overall response rate was low ( $8 \%$ of active CUA members). The small sample size is a limitation of the present study and likely reflects the lack of knowledge and experience in clinical treatment of hypogonadism. Future research should aim for a larger sample of respondents to increase the generalizability of results. Furthermore, it would be valuable to investigate potential differences between academic and community-based clinical practice patterns; future research should address this.

In summary, our survey was designed to assess urologists' "comfort zone" in prescribing testosterone replacement to hypogonadal men (not castrate) with a history of active or treated PCa. We found that the majority of urologists in this survey presently believe that TRT is safe in men who have had curative therapy for PCa, whether it be in the form of radical prostatectomy, radiation therapy, or brachytherapy. A lesser majority (20-30\% less) believe it is safe for those who are on active surveillance for low-grade PCa. But there exists a discrepancy between physicians' beliefs and their clinical practice patterns; a small cohort of urologists may have been accepting of the potential safety of TRT, but were still not comfortable carrying out this treatment in real-life practice. Perhaps they did not have men with both lowgrade $\mathrm{PCa}$ and testosterone deficiency in their practices or they were not screening for testosterone deficiency in their patients on active surveillance. Patient preference may also have played a role in low prescribing rates.

Findings from this survey support the creation of a nationwide database of men with PCa on TRT, as recommended by Morales. ${ }^{14}$ In the absence of a prospective, randomized, controlled trial, this type of a prospective registry would provide information to bolster the global body of evidence supporting TRT in men with PCa and impact more definitively on the standard of care.

Competing interests: The authors declare no competing personal or financial interests.

This paper has been peer-reviewed. 


\section{References}

1. Harman SM, Metter EJ, Tobin JD, et al. Longitudinal effects of aging on serum total and free testosterone levels in healthy men. J Clin Endocrinol Metab 2001;86:724-31. http://dx.doi.org/10.1210/ jcem.86.2.7219

2. Haas GP, Delongchamps N, Brawley OW, et al. The worldwide epidemiology of prostate cancer: Perspectives from autopsy studies. Can I Urol 2008; 15:3866-71.

3. Bassil N, Alkaade S, Morley JE. The benefits and risks of testosterone replacement therapy: A review. Ther Clin Risk Manage 2009;5:427-48.

4. Huggins $C$, Hodges CV. Studies on prostatic cancer. I. The effect of castration, of estrogen and of androgen injection on serum phosphatases in metastatic carcinoma of the prostate. Cancer Research 1941;1:293-7.

5. Agarwal PK, Oefelein MG. Testosterone replacement therapy after primary treatment for prostate cancer. J Urol 2005;173:533-6. http://dx.doi.org/10.1097/01.ju.0000143942.55896.64

6. Kaufman JM, Graydon RJ. Androgen replacement after curative radical prostatectomy for prostate cancer in hypogonadal men. J Urol 2004;172:920-2. http://dx.doi.org/10.1097/01.ju.0000136269.10161.32

7. Pastuszak AW, Pearlman AM, Lai WS, et al. Testosterone replacement therapy in patients with prostate cancer after radical prostatectomy. J Urol 2013;190:639-44. http://dx.doi.org/10.1016/i. juro.2013.02.002

8. Sarosdy MF. Testosterone replacement for hypogonadism after treatment of early prostate cancer with brachytherapy. Cancer 2007;109:536-41. http://dx.doi.org/10.1002/cncr.22438

9. Morales A, Black AM, Emerson LE. Testosterone administration to men with testosterone deficiency syndrome after external beam radiotherapy for localized prostate cancer: preliminary observations. BJU Int 2009;103:62-4. http://dx.doi.org/10.1111/i.1464-410X.2008.07882.x

10. Pastuszak AW, Pearlman AM, Godoy G, et al. Testosterone replacement therapy in the setting of prostate cancer treated with radiation. Int I Impot Res 2013;25:24-8. http://dx.doi.org/10.1038/iiir.2012.29

11. Morgentaler A, Lipshultz LI, Bennett R, et al. Testosterone therapy in men with untreated prostate cancer. J Urol 2011;185:1256-61. http://dx.doi.org/10.1016/i.juro.2010.11.084

12. Morgentaler A, Traish AM. Shiffing the paradigm of testosterone and prostate cancer: The saturation model and the limits of androgen-dependent growth. Eur Urol 2009;55:310-20. http://dx.doi.org/10.1016/i. eururo.2008.09.024

13. Kava BR. To treat or not to treat with testosterone replacement therapy: A contemporary review of management of late-onset hypogonadism and critical issues related to prostate cancer. Curr Urol Rep 2014; 15:422-014-0422-5.

14. Morales A. Effect of testosterone administration to men with prostate cancer is unpredictable: a word of caution and suggestions for a registry. BJU Int 2011;107:1369-73. hittp://dx.doi.org/10.1111/j.1464410X.2011.10193.x

Correspondence: Dr. Dean Elterman, Division of Urology, Department of Surgery, University Health Network, Toronto, 0N, Canada; dean.elterman@uhn.ca

\section{Indication and clinical use:}

Zoladex ${ }^{\circledast}$ is indicated for the palliative treatment of patients with hormone-dependent advanced carcinoma of the prostate (Stage M1 or Stage D2) and for use in combination with a non-steroidal antiandrogen and radiation therapy for the managementwof locally advanced $(\mathrm{T} 3, \mathrm{~T} 4)$ or bulky Stage T2b, T2c carcinoma of the prostate. Zoladex ${ }^{\circledR}$ can be used as adjuvant hormone therapy to external beam irradiation for patients with locally advanced prostate cancer (Stage T3-T4).

Treatment with Zoladex ${ }^{\circledR}$ and a non-steroidal antiandrogen should start 8 weeks prior to initiating radiation therapy and continue until completion of the radiation therapy. The safety and effectiveness of Zoladex ${ }^{\circledast}$ in children has not been established.

\section{Contraindications:}

- Hypersensitivity to goserelin/depot or any component of the container

Most serious warnings and precautions: Osteoporosis: Assessment of osteoporosis risk and management according to clinical practice and guidelines should be considered.

Tumor flare reaction: Patients at risk of developing ureteric obstruction should be closely monitored during the first month of therapy. Patients with vertebral metastases who are thought to be at particular risk of spinal cord compression should be closely monitored during the first month of treatment.

\section{Other relevant warnings and precautions:}

- Transient elevation of serum testosterone concentrations

- Increased cardiovascular risk factors

- Induced hypogonadism

- Impaired glucose tolerance

- Anemia

- Depression (sometimes severe)

- Pituitary-gonadal suppression

- Use in children has not been established; labeling reflects safety and effectiveness in patients over 65 years of age

- Treatment requires routine monitoring, physical examinations and appropriate laboratory tests

\section{For more information:}

Please consult the Product Monograph for Zoladex ${ }^{\circledast}$ at www.azinfo.ca/zoladex/pm965 for important information relating to adverse reactions, drug interactions and dosing information. The Product Monograph is also available by calling us at 1-800-565-5877.

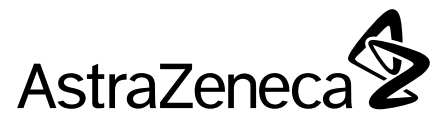

AstraZeneca Canada Inc. 1004 Middlegate Road, Unit 5000 Mississauga, Ontario L4Y 1M4. Zoladex and the AstraZeneca logo are registered trademarks of the AstraZeneca group of companies. (c) AstraZeneca 2015 